\title{
Understanding "Cool" in Human-Computer Interaction Research and Design
}

\author{
Dimitrios Raptis, Jesper Kjeldskov, Mikael Skov \\ Research Center for Socio+Interactive Design, Department of Computer Science, \\ Aalborg University, Selma Lagerlöfs Vej 300, \\ 9220, Aalborg, Denmark. \\ \{raptis, jesper,dubois\}@cs.aau.dk
}

\begin{abstract}
Recently a discussion has been initiated on what is cool and how HCI can use the concept in practice and design for it. This paper aims to provide a better understanding on cool as a concept from a theoretical and a practical perspective. From the theoretical perspective, we selected the HCI papers that focus on cool and we present their core findings. Then we performed a literature review on the concept of cool and we have identified its fundamental characteristics, through cool personalities and cool styles. From a practical perspective, we have studied how other domains have managed to successfully produce cool objects and we provide four suggestions on how to design cool digital artifacts. Finally, in this paper we also identify possible research directions in relation to cool, which if we manage to address we can increase our understanding on what is user experience and this can lead to the creation of better digital artifacts. Overall, this paper is a contribution towards researching and designing for cool, a research topic, which we believe it will initiate fruitful discussions in the HCI field.
\end{abstract}

\section{Author Keywords}

Cool; cool personality; cool style; user experience; design; authentic; rebellious; antisocial; exclusive; pleasure; social context;

\section{ACM Classification Keywords}

H5.m. Information interfaces and presentation (e.g., HCI): Miscellaneous.

\section{INTRODUCTION}

What is cool? Why we are referring to cool movies, cool people, or cool objects almost everyday? Are there some specific attributes that we perceive on an artifact and we characterize it as cool? If yes, can we define them? Why is cool relevant to $\mathrm{HCI}$ ?

$\mathrm{HCI}$ is studying our interactions with digital technologies and researches our experiences with them. User experience (UX, Hassenzahl and Tractinsky, 2006) is one of the main research streams of HCI and it encompasses the idea that our interactions with digital artifacts transcend effectiveness and efficiency. Although the

Permission to make digital or hard copies of all or part of this work for personal or classroom use is granted without fee provided that copies are not made or distributed for profit or commercial advantage and that copies bear this notice and the full citation on the first page. To copy otherwise, or republish, to post on servers or to redistribute to lists, requires prior specific permission and/or a fee.

$\mathrm{OzCHI} 13$, November 25 - 29 2013, Adelaide, Australia

Copyright 2013 ACM 978-1-4503-2525-7/13/11 ...\$15.00. usability literature also moved towards this direction with user satisfaction as a flag concept, UX studies our experiences from a broader perspective by encompassing concepts such as affect, emotion, hedonic and pragmatic qualities, fun, flow, enchantment, etc. Despite the fact that there is a significant amount of research directed towards understanding these concepts, there still are challenges that HCI needs to overcome. The most important ones are derived from the fact that we do not know if these concepts are enough to describe UX because the relations among them have not been studied in detail (Bargas-Avila and Hornbæk, 2011; Bargas-Avila and Hornbæk, 2012).

From a practical perspective all this research effort towards understanding user experience is being applied in order to produce better digital artifacts and HCI has managed to successfully provide the practitioners with valuable knowledge on how to do so. At the same time, an interesting fact is that many of the people that practitioners are designing for are using cool to describe their extraordinary interactions with the world: "My car is cool", "Her boyfriend is cool", "Skydiving is cool", etc. As a result, cool is a concept that is deeply rooted to the everyday life of our subjects and therefore we believe it will be beneficial both for researchers and practitioners to understand why they perceive an object, a person, or an activity as cool.

Consequently, we see a direct relation between HCI and the concept of cool both on a theoretical and on a practical level. Therefore, we propose that by researching on cool, HCI will be able to establish a deeper understanding on our users' experiences with digital artifacts, produce appropriate tools to guide practitioners to design for cool and thus significantly contribute in the creation of better digital artifacts. As a result, in this paper our research aim is to operationalize cool, by understanding what it is and by proposing suggestions on how to use it in practice.

In the rest of the paper we initially discuss how cool has been used in HCI up to now and then we present the results of a literature study on cool where we have identified its basic characteristics. Then we discuss the practical implications of the concept of cool and we present some suggestions on how our findings can be applied from practitioners in order to increase the coolness of their designs. Finally, we conclude our paper by suggesting future research directions. 


\section{COOL IN HCI}

We have searched in the literature in order to locate studies that dealt with the concept of cool, both in HCI and IT in general. The vast majority of the papers that use cool they don't refer to it as a concept, but they use it to attract more attention. Towards this end we may find papers that use the word cool as name for a programming language (e.g. Chandra et al., 1994).

Recently though, the HCI community touched upon cool as a concept and is tentatively discussing its implications. Holtzblatt et al. (2010) provided us with one of the first attempts to introduce cool to HCI and she presented a list of challenges for cool. We have summarized those challenges into:

- What? In order to design for cool we need to know what is it and understand its fundamental characteristics.

- Which? Which products are perceived as cool and how can we identify them?

- How? How can we design for cool?

Most of the related work that deals with cool as a concept contributes to what is cool. In Read et al. (2011) and Read et al. (2012) the authors focus on teenagers and they present us with the hierarchy of Cool: "Being Cool", by "Doing Cool Things" and by "Having Cool Stuff". They argue that very few people are actually and genuinely cool and the rest of the people eventually follow the trends they introduce by "Doing cool Things" and by "Having Cool Stuff". According to the authors, this creates opportunities and a design space, which HCI can take advantage in order to produce cool artifacts and applications. They also argue that cool is defined by six essential categories: rebellious, antisocial, retro, authentic, rich and innovative (Read et al., 2011) and the significance of these cool categories was explored by McCrickard et al. (2012). A different approach regarding what is cool comes from Holtzblatt (2011) where she focuses on cool experiences that bring joy in our lives and contribute to our personal motivations for life (accomplish, connection, identity and sensation). Furthermore, she presents an interesting discussion on how to design for joy and how to put it into action. Finally, there are two papers that present us with techniques on how to identify cool, like the "Cool Card Sort" (de Guzman, 2012) and the "Cool Wall", for collecting insights into cool preferences among teenagers (Fitton et al., 2012a; Fitton et al., 2012b).

All these papers constitute the initial attempts to introduce the cool concept to HCI. Since the volume of research on cool is rather low at this moment, most of the identified challenges are partially addressed. Through this paper we aim to contribute towards overcoming these challenges and in the following sections we present our own findings on what is cool as they emerged from a literature review, and we provide practical suggestions both on identifying which artifacts are perceived as cool and on how to design cool artifacts.

\section{WHAT IS COOL?}

We have managed to identify three different perspectives on who introduced the concept of cool for the first time. Southgate (2003) argues that Aristotle was the first one that introduced coolness in his book "Nicomachean Ethics”. Pountain and Robbins, (2000) and Gioia (2009) argue that coolness was defined in Renaissance Italy by Bardessare Castiglione in his "Book of the Courtier" (1516) where he proposed sprezzatura as a way of life (suggesting that we have to cultivate an appearance which allows us to be perceived as doing extremely difficult actions, effortless). Finally, some suggest that coolness was initially performed by African warriors as a way to appear detached in the face of danger (Pountain and Robbins, 2000; Thompson, 1973; MacAdams, 2001).

Independently from its origin, modern cool (cool as we know it today) appeared among black slaves in USA and it was slowly adopted by the majority of black individuals as a "strategic style" that allows black people to tip society's imbalanced scales in their favor (Majors and Billson, 1993) and to potentially transcend oppressive conditions and express themselves as men (Majors, 2001). Contemporary cool (cool as a characteristic of counterculture) became the means that small groups of black Jazz musicians resisted against the dominant white culture by encompassing a different way of dressing and behaving. This rebellious approach both against the dominant white culture and the old-fashioned way of performing Jazz, urged a number of Jazz musicians like Charlie Parker, Dizzy Gillespie, Thelonious Monk, Billie Holiday, etc., to contribute to a new, cooler style of Jazz: Bebop. The same essential elements that created Bebop appeared also in writing, with Beat authors like Jack Kerouac, painting with Jackson Pollock and later with Andy Warhol, and theatre with the creation of the "Living Theatre" from Judith Malina and Julian Beck (MacAdams, 2001).

Slowly cool transformed from a characteristic of people that belonged to counterculture (contemporary cool) into a significant attribute of mainstream culture (modern cool) and it still affects mass society through music, movies, books and products in general. As a result, this characteristic of a small rebellious community, it is now an attitude that is shared by most young people, and to certain extent by the parents as well (Poynor, 2000). At this moment we have to stress that cool was not a purely American phenomenon, as we observe similar transformations in other places too, for example in UK (Levy, 2002).

In our everyday life we use cool to describe people, objects and activities. When we assign the word cool, for example to an object, we basically perceive some of its characteristics as cool at a specific moment and inside a social context (Figure 1). If the context changes then the same characteristics can be perceived as uncool. As a result there is interplay between an individual and an object, inside a social context. 


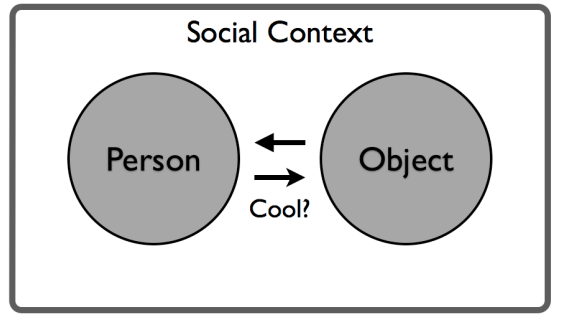

Figure 1: Person-object interplay inside a social context.

The first fundamental issue with this interplay is to understand if cool describes a set of characteristics that belong to the object:

"Cool is a quality of people, not of objects. Objects can only said to be cool as much as cool people use them."(Southgate, 2003)

"Cool is not something that inheres in artifacts themselves, but rather in people's attitude to them." (Pountain and Robbins, 2000)

From these quotes it is evident that there are not specific characteristics in an object that constitute it as cool. Pountain and Robbins (2000) and Southgate (2003) argue that cool is a property of the individual (it is a personal attitude) and inside a certain social context some of the object's characteristics are perceived as cool. If hypothetically the color black is cool when we observe it on a very expensive car that does not mean that all black objects will be perceived as cool too. Furthermore, Pountain and Robbins (2000) and Southgate (2003) provide us reasoning on how this cool perception is created: if cool people decide that an object is cool then the majority of the rest of the people will eventually perceive it as cool too.

"Cool is a phenomenon that we can recognize when we see it." (Pountain and Robbins, 2000)

Another fundamental characteristic of this interplay is that we instantly know if an object, a person, or an activity is cool the moment we see it (Pountain and Robbins, 2000; Gladwell, 1997). As a result, we don't need to think which are the characteristics of an object we perceive as cool; we instantly decide on that. The perception of cool is immediate.

"Cool is an expression of a community." (Thompson, 1973)

"Coolness is a set of shared meanings (e.g. language, self-presentation, artistic expression, values, attitudes) within a peer group which signify group affiliation."

(O’Donnell and Wardlow, 2000)

"Cool is a militant act, a way of staying below the radar screen of the dominant culture without loosing the respect of one's peers." (MacAdams, 2001)

Finally, the third characteristic of this interplay is related to social context, or the set of shared meanings (O'Donnell and Wardlow, 2000). The social context that influences the perception of cool is decided, defined and reshaped inside a group of peers. Individuals are cool only if they are accepted and bestowed by their peers (Connor, 2003; MacAdams, 2001; Gioia, 2009) and the same stands for the objects that the group accepts as cool. The groups identify themselves both by adopting a unique manifestation on what is cool (O'Donnell and Wardlow, 2000) and also by their strong rejection of what is not cool (Bird and Tapp, 2008). Consequently, the constant changes that happen inside a group in relation to what is cool or not act as a mechanism that ties a group together and demonstrates its distinctiveness from the rest of the groups and the mainstream culture (Saxton, 2005). Furthermore, this process of defining what is cool is dynamically constructed and constantly negotiated inside the group (Rodkin et al., 2006).

From our literature study it was evident that cool can be decomposed into (Figure 2): Cool personalities and cool styles (or inner cool and outer cool respectively, according to Nancarrow et al., 2001). Cool personalities possess some specific characteristics that make them cool and they are expressed by adopting a cool style. Although MacAdams (2001) argues that cool personality and cool style are inseparable we will use this distinction, for the purpose of this paper, as a way to decompose cool and we will provide details on our findings regarding the cool personality and the cool style in the following sections.

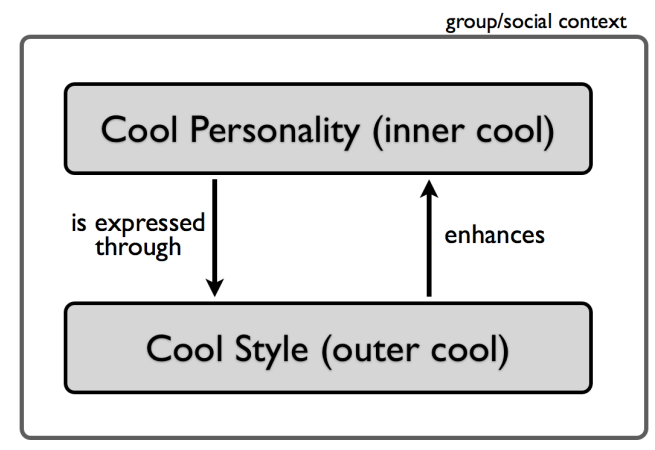

Figure 2: Cool personality and cool style.

\section{THE COOL PERSONALITY}

Although the perception on what constitutes a cool personal style may change rapidly, even during short periods of time, there are specific attributes that characterize a cool personality, which remain constant and independent from the style they currently adopt. In the following paragraphs we will present these characteristics.

"Cool is a permanent state of private rebellion." (Pountain and Robbins, 2000)

Pountain and Robbins (2000) argue that a cool personality is characterized by a permanent state (permanent because when someone enters this state it never leaves) of private rebellion (private because cool is not transformed to a mass, group resistance to any kind of authority and/or the conformity imposed by the mainstream culture, but it instead remains a personal "battle" characterized by detachment and irony). The authors move also one step further and they propose that the cool personality is defined by three personality traits: 
narcissism, ironic detachment and hedonism (Pountain and Robbins, 2000). In fact during the 50's cool individuals embraced an apolitical stance in favor to personal development and pleasure seeking that can be observed even today (Pountain and Robbins, 2000; MacAdams, 2001). Furthermore, this pleasure seeking behavior can be so profound that it may reach the level of self-destructiveness and even flirting with death and it is often related to "live for today" philosophy (Pountain and Robbins, 2000). Additionally, this strong focus on personal development and pleasure inside a group of peers is also expressed by the individual's resistance to anything related to the norms imposed by society, or any value that contradicts the values of the group. Thus, cool personalities express a high level of antisocial behavior.

"Coolness means poise under pressure and the ability to maintain detachment, even during tense encounters." (Majors and Billson, 1993)

At the core of the cool personality lies the ability to appear emotionally neutral, disengaged and nonchalant (Stearns, 1994). The cool personalities are detached and maintain this detachment and the sense of being-incontrol in every situation they might encounter. To do so they adopt a calm, ironic pose that enhances the perception of having things under control (Pountain and Robbins, 2000; MacAdams, 2001). Through this way, cool acts as a mask, or as an emotional mantle (MacAdams, 2001; Stearns, 1994). No matter how difficult an unexpected situation might be, or how dangerous an action might be one should maintain his/her cool and his/her emotional neutrality and posture without showing any feelings, such as anger, or fear, and it does not matter if $\mathrm{s} /$ he does the right, or wrong thing as long as it is done the right way (MacAdams, 2001). Furthermore, besides unexpected situations, cool is strongly related to the way expected/normal activities are performed. Activities, that are usually difficult to perform, must appear as trivial and easy. The more easy they appear the more perfect they look and the cool personalities appear in control, as "knowing what they are doing". Instances of such attitude can be found in sports, when for example Michael Jordan got his "Air" nickname, because he was cool enough to be perceived as walking on air while playing basketball (Pountain and Robbins, 2000; Majors, 2001). Furthermore, the cool personality expresses this behavior not only in difficult/dangerous situations, but also general in life (Moore, 2004; Nancarrow et al., 2001).

"Authenticity is the truest hallmark of cool behavior." (Southgate, 2003)

Cool is strongly related to authenticity. Cool personalities resist and oppose to anything that they consider as fake, or copied. They perceive music, books, cars, etc., as cool as long as they are authentic, out of the ordinary and part of counterculture. But even these cool objects, or activities become uncool as soon as they enter the mainstream culture, since they become unauthentic and corrupted (Pountain and Robbins, 2000). Taking this attitude into consideration there is a strong relation between cool and innovation, since cool personalities are excited with novel things and constantly seek things that challenge the mainstream (Pountain and Robbins, 2000).

"Cool itself is intrinsically judgmental and exclusive."

(Pountain and Robbins, 2000)

The cool personalities are judgmental towards the rest of the world, or the "sheep" (Nancarrow et al., 2001), which do not know what is cool at the moment and follow what the mainstream culture dictates. They are also highly confident that they know what is cool (Charles, 2002) and they find satisfaction through exclusive objects or activities that the "sheep" do not know: for example by paying a visit to an unknown bar.

In summary, cool is a personal attitude toward objects, people and activities from cool personalities which are characterized as rebellious against the dominant culture and any form of authority (ranging from parents to governments), antisocial, always trying to appear in control in every situation, making difficult activities to become/appear as easy, embracing authenticity and exclusivity and strongly tied to a group of peers, while investing in personal development and pleasure by projecting an emotional neutrality. Table 1 summarizes the fundamental characteristics of cool personalities.

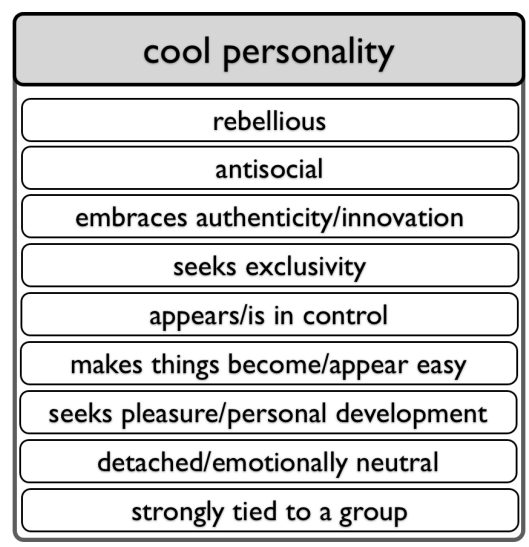

Table 1: Cool personality characteristics.

\section{THE COOL STYLE}

Through our review on cool it was evident that cool is not a set of characteristics that belong to an object. In fact cool is an attitude that belongs to people and is demonstrated through style. According to Gioia (2009) a cool style is characterized by somebody's attire, accessories, language and pose, which are cool only if they are accepted by one's peers. One's attire, language and pose are elements of one's personality, but accessories are more related to his style (even though this style is defined by the cool personality). Cool and style are inseparable (MacAdams, 2001) and thus in order to design cool digital artifacts it is important to design artifacts that concurrently match a cool personality and fit a cool, personal style. The difficulty in this approach is that cool style is always evolving and changing, influenced both by the cool personality and the social context. Therefore we argue that in order to define a cool style we must describe the extreme forms that it can take. We have applied this approach to our literature study and 
a set of cool style characteristics have emerged. In the following paragraphs we will present our findings and we will demonstrate them through examples.

Cool style is: minimalistic.......flamboyant

Cool style can be instantiated both in a minimalistic, or a flamboyant form (or elegant/gigantic according to Pountain and Robbins, 2000) depending on the social context and the perception of cool that a group of peers has. A typical example can be found in clothing. While on one hand some people adopt a style that focuses on elegance and minimalistic-simplistic elements (for example black tie suits), on the other hand we might see groups of people that prefer flamboyant clothing with flashy details that immediately attract attention (for example hip-hop buggy pants and gigantic gold chains, or "rock" leather jackets covered with steel nails). Thus, at the same time two different groups of people (or even the same group if we observe how differently Bebop musicians were dressed) are using extremely different accessories that they perceive as cool: flashy, flamboyant ones, or minimalistic and elegant.

\section{Cool style is: expensive........cheap}

The cool personality is related to authenticity, exclusivity and rebelliousness. In order to express these characteristics though a cool style, individuals usually acquire specific objects that can be either expensive, or cheap. A typical example of an expensive cool style can be found in cars: Ferrari's, Porsche's and other expensive cars are perceived by most people as cool, since they are exquisite and very difficult for common people to acquire. On the other hand, authentic and exquisite objects can also be cheap, as for example an old VW Beetle, which was transformed from an "ugly" German car to a symbol of counterculture (Imseng, 2011). Another example of this duality can be also found in shoes, where for example, it is currently considered as cool to wear expensive clothes with sneakers, such as All Star shoes, which is a cheap, but nevertheless a cool item.

Cool style is: beautiful.......ugly

Cool style can be beautiful, shining and adorable and cool can be ugly and dark. Both style instantiations can appeal even to the same people in different contexts. A typical example can be found in music. While many teenagers enjoy hearing melodic, pop songs from artists like Adele, at the same time we currently observe the dominance of another, more dark and more "ugly" style of music, which is also perceived as cool: dubstep. One of the dubstep definitions provided by Urban online Dictionary is: "The purest form of musical crack cocaine; most effective when ingested intra-subwooferly. Found almost universally at dance parties attended by cool people everywhere". For many, dubstep is ugly, violent and not even music, but for the cool personalities it is the ultimate vehicle for personal expression, in a similar way as Rave, or Grunge music was during the ' 90 s. Towards this end, a known example of a whole generation intentionally adopting ugly stuff as a way to rebel against the dominant culture can be found during the ' $90 \mathrm{~s}$ among the Grunge generation. Grunge style adopted the ugliest clothes there could be found on every shop in order to project its revolt against marketing (Rushkoff, 2008). This relation also to "ugliness" is also demonstrated by the usage of the word "sick" to describe something excellent and wonderful, which entered the mainstream slang at about the same time as grunge music, or the use of the word "wicked" with the same connotation previously (Pountain and Robbins, 2000).

\section{Cool style is: innovative........retro}

Cool adores innovation and new exciting stuff and anything that is moving towards the future (MacAdams, 2001). A cool style can adopt the latest technological innovations in order to stand out from the masses and the rest of the people who do not know what is cool yet. A typical example of considering new technologies as cool can be found in the adoption of Apple products, especially on the early days where the company was unknown to the majority of people. At the same time we observe that some groups of individuals are opting for retro objects that clearly signify that they come from another age (Nancarrow and Nancarrow, 2007). Thus, for example, we can observe an increase in usage of old cell phones (Kirman, 2012), which are primitive in comparison to the modern ones, but nevertheless their retro characteristics are perceived as cool. The same tendency towards retro can be found also in clothing with a booming market that deals with vintage products, or even in computer gaming where there are specific communities that focus on playing, maintaining and expanding old computer games, despite the fact there are modern versions of them (for example the Championship Manager 2001/2002 online community).

\section{Cool style is: illicit.......licit}

Cool personalities seek pleasure and in this quest they often reach the level of self-destructiveness because pleasure can sometimes found to things/activities that are not legal and thus they often flirt with criminality and danger (Pountain and Robbins, 2000; Gioia, 2009; MacAdams, 2001). For example, it is a fact that many Bebob Jazz musicians were excessively using heroin (MacAdams, 2001). Nevertheless, this interplay between licit and illicit is inherent in a cool style, with one requirement: flirting with criminal behavior is cool, as long as the individual is in control of the situation; then it is not cool anymore. For example there are research data that children characterize as cool their peers that are at the same time popular and aggressive (tough) (Rodkin et al., 2006). Those individuals according to Horton et al. (2012) are perceived as cool as long as they lie in zone of non-harmful rebelliousness and they become uncool when they reach the level of criminal rebelliousness. A typical example of this adoration on illicit objects can be also found in music video clips where "gangsta-rap" is enhanced by showing off guns. On the other hand, licit objects and activities can of course be considered as cool, as long as they match the style of the individual.

Table 2 summarizes the different characteristics that a cool style may have. 


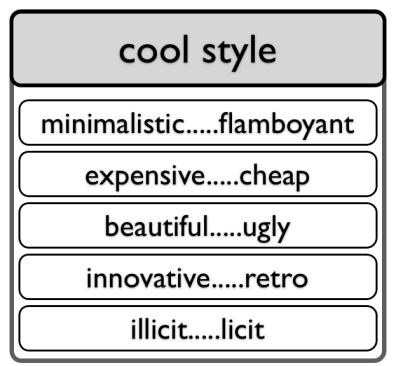

Table 2: Cool style characteristics.

\section{COOL IMPLICATIONS}

Up to this point we focused on what is cool and through a literature study we identified that cool is a property of people and not of objects and we also presented the fundamental characteristics of cool personalities and cool styles. In the following subsections we will apply a more pragmatic approach and discuss how practitioners can take advantage of the concept in order to design for cool.

Let's imagine a scenario where a group of designers and developers wants to create an application, which will be perceived as cool. The first thing they need to do is to understand what is cool and which of its characteristics can be included to their design. Since designers often use existing artifacts as inspiration, then in order to find out how to instantiate these characteristics it is important to have an objective way to understand which of the existing artifacts are perceived as cool.

\section{Which artifacts are perceived as cool?}

In order to answer which of the existing digital artifacts are perceived as cool practitioners and researchers need to understand how cool is created in general.

The first step in the creation of cool comes from cool personalities, which are the Innovators (Taylor, 2009) and they are about $2-3 \%$ of the population (The Merchants of cool, 2001). They either envision something new and innovative (Frank, 1997), or they personalize existing objects and make them cool (MacAdams, 2001). Thus, innovators are the ones that create and identify cool (Gladwell, 1997; MacAdams, 2001). The objects the Innovators use and the activities they do, are adopted by the Trendsetters (the ones who mimic Innovators), then the process continues with the Early Adopters, and finally objects and activities become part of the mainstream culture (Taylor, 2009; The Merchants of Cool, 2001). Cool is adopted by one group after the other and in this specific order (Rogers, 2003). As soon as these objects reach the mainstream they are immediately perceived as uncool by the Innovators (Gladwell, 1997). At this moment the rest of the people still perceive them as cool, but most of them will eventually perceive them as uncool in the end. Very few of these objects though reach the level of universally cool (Schiller, 2012), or classic (Nancarrow et al., 2001) and even though they are absorbed by the masses, they are still perceived as cool by the majority of people. Such examples are Ray-Ban sunglasses, or Absolut Vodka (The Merchants of Cool,
2001). Figure 3 depicts the different transformations of an object in relation to cool.

From Figure 3 it is evident that in order to identify which objects will be perceived as cool by the mainstream, we need to identify which objects Innovators perceive as cool at the moment. If we consider we know from the literature that people select specific products because they project elements of their personality (Jordan, 1997; Phau and Lau, 2001), then we propose that our identified cool personality characteristics can be used as the starting point for locating which of the digital artifacts are perceived as cool by the Innovators. Thus, if Innovators perceive a digital artifact as rebellious, exclusive, authentic and projecting some or all of the characteristics of the cool personality, then the rest of the people will also perceive it as cool after some time. The challenge for practitioners is that they need to be careful on who they ask to assess the coolness of an artifact as different types of people will give different responses depending if they are Innovators, or not. A tool for locating Innovators is the Trendsetting Questionnaire (TDS-K) from Batinic et al. (2008).

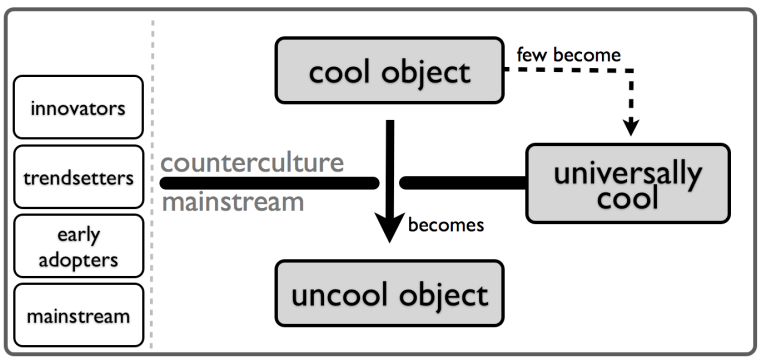

Figure 3: Cool transformations.

Towards this end, HCI should focus on providing the practitioners with the necessary instruments to objectively measure the coolness of existing artifacts. If we consider that cool is a personal attitude and that there are many available techniques in measuring attitudes (Oppenheim, 2001), we argue that HCI should aim in producing an instrument to measure perceived coolness. We believe that our identified cool personality characteristics can contribute to the creation of this instrument and until it is produced they can also guide practitioners into defining possible cool artifacts. For example we believe that such candidate cool artifact is Ekkomaten (Basballe, 2012), which is a digital, historical guide for the Danish city of Aarhus that seems to violate many established design guidelines: it is bulky, unconventional, some can even say ugly. Yet, interacting with it resulted in exciting experiences, especially for all of us who became familiar with it during NordiCHI 2012 conference. We can assume that Ekkomaten was perceived as cool as it was rebellious in comparison to typical digital guides, authentic, exclusive, it allowed its users to be in control and its style was flamboyant, retro and somehow ugly. Of course more research data are needed for concrete results, but nevertheless by using the cool personality and style characteristics practitioners can have an indication on which artifacts are perceived as cool. 


\section{How can we design for cool?}

The third challenge is how to approach the cool concept as a practitioner and use it in order to produce artifacts that are perceived as cool. In relation to cool we believe that practitioners:

1. Can create inceptive cool experiences by designing innovative artifacts that do not exist before, or by envisioning new uses for an artifact that nobody had thought before.

2. Can transform existing artifacts by cooling them up (make them perceived as cool).

In the following subsections we present our suggestions on these two types of design activities.

\section{Creating inceptive cool experiences}

Creating inceptive cool experiences is the most challenging task for practitioners, because it is a question that has broader implications that only designing for cool. We are basically asking how to create disruptive technologies (Christensen, 1997), which permanently change the way we experience the world. For example, how can we produce digital artifacts that create the same cool feeling as holding an iPad for the first time? If we try to evaluate the iPad in relation to cool we will observe that when it was released: it was authentic, rebellious, exclusive, it allowed its users to be in control and to appear as making difficult tasks as easy and it gave pleasure to its owner. Additionally, it was instantiated through a minimalistic, expensive, beautiful and innovative style. Of course some might say that our cool personality and cool style characteristics are easier to apply in order to evaluate something that already exists, than using them to design something new. We agree that they do not constitute a design method for cool, but we believe that they create a design space that cool can emerge. At the same time, we suggest that practitioners should take advantage of a core characteristic of the cool personality: it's tight relation to a group of peers. We propose that in order to produce inceptive cool experiences with digital artifacts practitioners need to study in detail the group of peers they are designing for and produce artifacts that have value and meaning (Hallnäs and Redström, 2002) for that specific group. In order to do this, it is important not only to be engaged to fieldwork but to do better fieldwork by increasing the depth and the duration of their field studies (Kjeldskov and Paay, 2012).

\section{Cooling existing artifacts up}

Let's imagine one of our typical users who owns a modern mobile phone and a tablet. We know from user experience literature that this user did not select these devices only because they are efficient and effective. He selected the specific devices because they offer to him something more: he perceives them as beautiful, desirable, cool. Furthermore, he usually applies the same approach throughout his everyday life and thus he does not buy the first pair of shoes he sees, but he selects the one pair that matches his style. As a result, we believe that creating inceptive cool experiences is equally important as cooling existing digital artifacts up and the challenge for practitioners and researchers is to transform the hundreds of the available digital artifacts that do not offer the added value that users enjoy in their personal life (for example the pleasure of having an iPad, or a cool pair of shoes) into more cool and desirable artifacts. Such examples of artifacts that need to be cooled up are the banking systems that often have anachronistic interfaces that each day thousands of employees interact with.

In order to understand the possible ways of cooling existing digital artifacts up, we believe that it is useful to become familiar with how other domains have managed to create objects that are perceived as cool. Since you cannot sell cool per se, but you can sell the "idea of cool" (MacAdams, 2001) as a label for fashionable hipness (Gioia, 2009), big brands and advertisement companies constantly pump into the mainstream what the Innovators perceive as cool, in order to enhance consumerism. For business, this quest of seeking for cool never ends (Gladwell, 1997; The Merchants of Cool, 2001) and in fact they are so successful that they manage to create objects that directly match a cool personality and fit inside a cool style. For example, during the 60's American business successfully offered the public authenticity, individuality, difference and rebellion (Frank, 1997). A typical example of such targeted consumerism are the products that take advantage of the "cool rebel" Ernesto Che Guevara: from cheap T-shirts to expensive Louis Vuitton handbags. Frank (1997) describes this process in detail and the same phenomenon is elegantly presented in the BBC4 documentary "The century of the Self" (Curtis, 2002).

Thus, the interesting question for practitioners is: if business managed to successfully cool things up, can they do the same? We propose four distinct approaches that practitioners can follow in order to design for cool: 1) focus on the Innovators, 2) focus on the mainstream, 3) focus on universally cool items, and 4) allow cool to emerge. In the following subsections we describe these approaches in detail.

\section{Focus on the Innovators}

We believe that the first approach that practitioners can adopt in order to produce artifacts that are perceived as cool is to do exactly what the business does and perform coolhunting. In order to grasp what Innovators perceive as cool businesses perform coolhunting, aiming to identify a subcultural phenomenon before it reaches the mainstream culture, in order to to be the ones that will take advantage of it (Frank, 1997; The Merchants of Cool, 2001). Basically, they observe what the Innovators are doing/wearing/listening at the moment and they incorporate these to their designs, as they know that the mainstream will eventually follow the Innovators. As a result, coolhunting is more about observing and incorporating and less about inventing (O'Donnell and Wardlow, 2000). The challenges though for successfully performing coolhunting are: a) only Innovators can identify/do cool (Gladwell, 1997; MacAdams, 2001) and thus practitioners need to include Innovators into their 
design teams, b) coolhunting is expensive (Bird and Tapp, 2008). Nevertheless, we believe that practitioners can invest more resources on coolhunting since: a) it can fit really well with the development circle of digital artifacts since it provides the necessary time to built an artifact, which will be perceived as cool by the mainstream when it will be released, and b) it is proven from other domains that it is a really successful technique in order to design for cool.

\section{Focus on the mainstream}

A second approach for practitioners on how to design perceived cool digital artifacts is not focus on Innovators through coolhunting but to observe objects, activities and people that are at this moment considered as cool by the mainstream. By excessively studying what the mainstream considers as cool at this moment practitioners can have a variety of inspirational ideas on which style their designs should adopt in order to be cooled up. For example, there is a large volume of literature (e.g. Evans, 2007 ) that presents to us cool cars, hotels, webpages, etc. We argue that during design sessions practitioners can identify specific design characteristics from those perceived cool objects and try to incorporate them to their designs. The disadvantage of this approach, though, is that it requires a fast development circle since the perception of what is cool can change rapidly. As a result this approach could be used in order to make minor adjustments, for example in the interface of an application.

\section{Focus on universally cool items}

Our third suggestion on how to design for cool is to compare our designs with universally cool, or classic objects. These objects share the common characteristic that they are perceived as cool by the vast majority of our subjects, independently from where they belong (mainstream or counterculture) and if they are Innovators or not. Thus, these objects somehow stand beyond the influences of social context and are perceived as cool almost universally. We propose that by asking their subjects to compare their designs with these universally cool items (an approach which can be really successful according to Oppenheim, 2001), practitioners will be able both to evaluate their designs in relation to cool, and to identify possibilities for new designs. In order to identify these universally cool items practitioners can apply the previously described approaches for finding which products are perceived as cool, but they should focus not only to Innovators, but to the majority of people.

\section{Allow cool to emerge}

Finally, our fourth suggestion is somehow the reversed from the previous three. Instead of trying to produce digital artifacts that will be perceived as cool, practitioners can provide the freedom to their users to personalize them. Thus, instead of trying to create a "cool jacket", they can allow the users to "attach pins to the jackets" they offer to them, and thus make them as cool as they perceive. For example, we know that many users personalize their mobile phones (Moggridge, 2007) in order to match their style. By allowing this freedom to exist in all levels of their digital artifacts (for example the interface of the applications) practitioners will not produce cool per se, but they will allow cool to be created by users.

\section{CONCLUSIONS}

In this paper we systematically decomposed the concept of cool through a literature review in order to contribute to the three challenges we have identified from related work: what is cool, which objects are perceived as cool and how we can design for cool. We approached cool from a cool personality and a cool style perspective and we have presented their fundamental characteristics. In summary, cool personalities (the Innovators) are antisocial, rebellious, seeking pleasure, personal development, innovation, authenticity and exclusivity, by appearing as being-in-control and as performing difficult tasks as easy, without showing emotions, while being strongly tied to a group. These personalities are expressed through a cool style, which can be minimalistic/flamboyant, expensive/cheap, beautiful/ ugly, innovative/retro and illicit/licit depending on the perception that their group of peers has in relation to cool. Furthermore, we have presented our suggestions on how these characteristics can be applied to practice in order to produce digital artifacts, which will be perceived as cool by focusing on Innovators, focusing on the mainstream, focusing on universally cool items and by allowing cool to emerge.

Overall, we believe that this paper contributes on having a better understanding of cool as a concept and we will conclude it with some possible research directions that we believe are interesting for HCI. Since cool has already been introduced to HCI then one of the first directions for research should be the investigation of cool's relation to user experience constructs (Bargas-Avila and Hornbæk, 2011; Bargas-Avila and Hornbæk, 2012). Is there any relation between cool and usability, cool and hedonic and pragmatic values, or cool and the rest of user experience constructs? For example, we know that beautiful objects are perceived as usable (Tractinsky et al., 2000), but are cool objects perceived as usable too? We strongly believe that such a research effort will be beneficial not only in understanding cool, but also in better understanding user experience.

Additionally, we believe that it is important to study in detail the effect of first impression in relation to cool. Since most of the literature agrees that we instantly decide if an object is cool and if we consider that HCI has research findings on the significance of first impression on user experience (for example Lindgaard et al., 2006, provided valuable data on the effect of first impression in relation to visual appeal), we propose that it is worth investigating the impact of first impression in relation to cool. By researching more on the subject we argue that we will be able to gain a deeper understanding on the parameters that affect user experience and we will be able to assist practitioners in taking into consideration these parameters during the development processes. 
Finally, we argue that it is important to investigate the fact that cool is strongly influenced by social context and as a result the perception of cool is so diverse in different groups, places, cultures and generations. We believe that by studying these different contexts we will be able to have a deeper understanding on the parameters that affect the perception of cool and we will provide better suggestions for design to practitioners.

Overall, we argue that cool is a valuable construct for HCI as it will provide us with new insights both on how to design better artifacts and on what is user experience. Since cool is making its initial steps into the HCI field we believe that a lot of research needs to be done, and we proposed some research and design directions that HCI could take. We suggest that our proposed cool personality and style characteristics can be the starting point towards these directions. As a future work we plan to continue researching on cool and start addressing the research and practical challenges we pointed out.

\section{ACKNOWLEDGMENTS}

The research behind this paper was partly financed by the Danish Research Councils (grant number 09-065143).

\section{REFERENCES}

Bargas-Avila, J.A., and Hornbæk, K. Old Wine in New Bottles or Novel Challenges? A Critical Analysis of Empirical Studies of User Experience. In Proc. CHI 2011, ACM Press (2011), 2689-2698.

Bargas-Avila, J.A., and Hornbæk, K. Foci and Blind Spots in User Experience Research. Interactions, 19, 6 (2012), 24-27.

Basballe, D.A., Breinbjerg, M., and Fritsch, J. Ekkomaten: an auditory interface to the 18th century city of Aarhus. In Proc. NordiCHI 2012, ACM Press (2012), 742-745.

Batinic, B., Wolff, H.G., and Haupt, C.M. Construction and Factorial Structure of a Short Version of the Trendsetting Questionnaire (TDS-K): A CrossValidation Using Multigroup Confirmatory Factor Analyses. European Journal of Psychological Assessment, 24, 2 (2008), 88-94.

Bird, S. and Tapp, A. Social Marketing and the meaning of Cool. Social Marketing Quarterly, 14, 1 (2008), 1829.

Championship Manager 2001/2002 online community: http:/www.champman0102.co.uk/

Chandra, R., Gupta, A., and Hennessy, J.L. COOL: An object-based language for parallel programming. Computer , 27, 8 (1994), 13-26.

Charles, G. Cool for Cash. Marketing Week, 25, 33 (2002), 24.

Connor, M.K. What is cool?: Understanding Black Manhood in America. Agate Publishing (2003), Chicago.

Christensen, C.M. The innovator's dilemma: when new technologies cause great firms to fail. Harvard Business School Press (1997), Boston, Massachusetts.
Curtis, A. The century of the Self. BBC 4 documentary (2002).

de Guzman, E.S. "Cool” Card Sort: Adapting Desirability studies to assess "Cool"-ness. ACM CHI Workshop on Cool aX Continents, Cultures and Communities 2012.

Evans, D. Coolhunting. A guide to high design and innovation. Southbank Publishing (2007), London.

Fitton, D., Horton, M., Read, J.C., Little, L., and Toth, N. Climbing the Cool Wall: Exploring Teenage Preferences of Cool. Ext. Abstracts CHI'12, ACM Press (2012), 2093-2098.

Fitton, D., Read, J.C., Horton, M., Little, L., Toth, N., and Guo, Y. Constructing the Cool Wall: A Tool to Explore Teen Meanings of Cool. PsychNology, 10, 2 (2012), 141-162.

Frank, T. The Conquest of Cool. Business Culture, Countercurture, and the Rise of Hip Consumerism. The University of Chicago Press (1997), Chicago.

Gioia, T. The birth and death of the Cool. Speck Press (2009), Golden Colorado.

Gladwell, M. The Coolhunt, New Yorker, March 17, 1997. Retrieved from: http://www.gladwell.com/1997/ 1997_03_17_a_cool.htm

Hallnäs L., and Redström J. From use to presence: On the expressions and aesthetics of everyday computational things. Transactions on Computer-Human Interaction, 9, 2 (2002), 106-124.

Hassenzahl, M. and Tractinsky, N. User Experience - a Research Agenda. Behaviour \& Information Technology, 25, 2 (2006), 91-97.

Holtzblatt, K., Rondeau, D.B., and Holtzblatt, L. Understanding "Cool". Ext. abstracts CHI '10, ACM Press (2010), 3159-3162.

Holtzblatt, K. What Makes Things Cool? Intentional Design for Innovation. Interactions, 18, 6 (2011), 4047.

Horton, M., Read, J.C., Fitton, D., Toth, N., and Little, L. Too Cool at School - Understanding Cool Teenagers. PsychNology Journal, 10, 2 (2012), 73-91.

Imseng, D. Think Small. The story of the world's greatest ad. Full Stop Press (2011), Uster, Switzerland.

Jordan, P.W. Products as personalities. In (ed.) M.A. Hanson, Contemporary Ergonomics. Taylor \& Francis (1997), 449-453.

Kirman, B. Hipsters, Trendies and Rebels: If Fun is Cool, is Game Design Cool Design? ACM CHI Workshop on Cool aX Continents, Cultures and Communities 2012.

Kjeldskov J., and Paay J. A longitudinal review of Mobile HCI research Methods. In Proc. MobileHCI 2012, ACM Press (2012), 69-78.

Levy, S. Ready, Steady, Go. Swinging London and the invention of Cool. Fourth Estate (2002), London.

Lindgaard, G., Fernandes, G., Dudek, C., and Brown, J. Attention web designers: you have 50 milliseconds to make a good first impression! Behaviour and Information Technology, 25, 2 (2006), 115-126. 
MacAdams, L. Birth of the Cool. Beat, Bebop, and the American Avant-Garde. The Free Press (2001), New York.

Majors, R., and Billson, J.M. Cool Pose: The Dilemmas of Black Manhood in America. Touchstone (1993), New York.

Majors, R. Cool Pose: Black Masculinity and Sports. In (eds) S.M. Whitehead and F. Barrett, The Masculinities Reader, Polity Press (2001), Oxford.

McCrickard, D.S., Doswell, F., Barksdale, J., and Piggott, D. Understanding Cool in Computing for AfricanAmerican Youth. ACM CHI Workshop on Cool aX Continents, Cultures and Communities 2012.

Moggridge, B. Interaction Design (IDEO). Innovation Forum 2007, Potsdam, Germany. Available from: http://interface.fh-potsdam.de/innoforum/english/ 09_videos.php [Accessed 31 May 2013]

Moore, R.L. We're Cool, mom and dad are swell: Basic slang and generational shifts in values, American Speech, 79, 1 (2004), 59-86.

Nancarrow, C., Nancarrow, P., and Page, J. An analysis of the concept of cool and its marketing implications. Journal of Consumer Behaviour, 1, 4 (2001), 311-322.

Nancarrow, C., and Nancarrow, P. Hunting for cool tribes. In (eds) Cova, B., Kozinets, R.V., and Shankar, A., Consumer Tribes, Butterworth-Heinemann (2007), 129-143.

O'Donnell, K.A., and Wardlow, D.L. A Theory on the origins of Coolness. Advances in Consumer Research, 27 (2000), 13-18.

Oppenheim, A.N. Questionnaire Design, Interviewing and Attitude Measurement. Continuum (2001), London.

Phau, I. and Lau, K. Brand personality and consumer self-expression: Single or dual carriageway? The Journal of Brand Management, 8, 6 (2001), 428-444.

Pountain, D., and Robbins, D. Cool Rules. Anatomy of an Attitude. Reaction Books (2000), London.
Poynor, R. Cool. Optic Nerve, 54, 3 (2000), 64-65.

Read, C.R., et al. Understanding and Designing Cool Technologies for Teenagers. Ext. Abstracts CHI '11, ACM Press (2011), 1567-1572.

Read, C.R., Horton, M., and Fitton, D. Being CoolGetting Persona-1. ACM CHI Workshop on Cool aX Continents, Cultures and Communities 2012.

Rodkin, P.C., Farmer, T.W., Pearl, R., and Van Acker, R. They're Cool: Social Status and Peer Group Supports for Aggressive Boys and Girls. Social Development, 15, 2 (2006), 175-204.

Rogers, E.M. Diffusion of Innovation, 5th ed. Free Press (2003), New-York.

Rushkoff, D. The Pursuit of Cool. (2008) Available online: http://www.rushkoff.com/articles-individual/ 2008/10/28/the-pursuit-of-cool.html

Saxton, G. Collections of Cool. Young Consumers, 6, 2 (2005), 18.

Schiller, J. Who is the coolest of them all. ACM CHI Workshop on Cool aX Continents, Cultures and Communities 2012.

Southgate, N. Coolhunting, account planning and the ancient cool of Aristotle. Marketing Intelligence \& Planning, 21, 7 (2003), 453-461.

Stearns, P.N. American Cool: Constructing a Twentiethcentury Emotional Style. New York University Press (1994), New York.

Taylor, M. The book of Cool. Running Press Book Publishers (2009), Philadelphia.

The Merchants of Cool. Frontline Documentary (2001). Available online: http:/www.pbs.org/wgbh/pages/ frontline/shows/cool/view/

Thompson, R.F. An aesthetic of Cool. African Arts, 7, 1 (1973), 41-91.

Tractinsky, N., Katz, A.S. and Ikar, D. What is beautiful is usable. Interacting with Computers, 13, 2 (2000), 127-145. 